2005, 109, 19553-19555

Published on Web 09/29/2005

\title{
Controlling the Length and Shape of Gold Nanorods
}

\author{
Hao Ming Chen,${ }^{\dagger}$ Hsin-Chieh Peng, ${ }^{\dagger}$ Ru-Shi Liu, ${ }^{*},{ }^{\dagger}$ Kiyotaka Asakura, ${ }^{\dagger}$ Chien-Liang Lee, ${ }^{\S}$ \\ Jyh-Fu Lee," and Shu-Fen $\mathrm{Hu}^{\perp}$ \\ Department of Chemistry, National Taiwan University, Taipei 106, Taiwan, Catalysis Research Center, \\ Hokkaido University, Sapporo, Japan, Materials Research Laboratories, Industrial Technology Research \\ Institute, Chutung 310, Taiwan, National Synchrotron Radiation Research Center, Hsinchu 300, Taiwan, and \\ National Nano Device Laboratories, Hsinchu 300, Taiwan
}

Received: July 4, 2005; In Final Form: September 14, 2005

\begin{abstract}
In this paper, we report a new approach to fabricate gold nanowires by controlling the volume of the growth solution. The shape evolutions ranging from fusiform nanoparticles to 1-D rods were observed. Increasing the addition of the growth solution can control the length of nanorods. The length of the rods can be extended to $2 \mu \mathrm{m}$, and nanorods with aspect ratios of up to $\sim 70$ could be obtained.
\end{abstract}

\section{Introduction}

Numerous characteristics of nanomaterials depend on size and shape, including their catalytic, optical, and physical properties. ${ }^{1-3}$ A number of chemical approaches have been actively explored to process metal into 1-D nanostructures. ${ }^{4}$ Gold nanorods have been synthesized by electrochemical reduction method in the presence of cetyltrimethylammonium bromide $(\mathrm{CTAB})^{2,5}$ and by seed-mediated growth in a surfactant template. ${ }^{3}$ The growth mechanism of 1-D gold nanoparticles in the presence of CTAB has been extensively investigated in the literature (e.g., growth direction, micelles properties, optical absorption spectra, and effect of $\mathrm{pH}) .^{6-10}$ Although monodispersed gold nanorods (average length of $\sim 500 \mathrm{~nm}$ ) had been synthesized with a three-step seeding method at room temperature, ${ }^{3 b}$ the actual reaction process of gold atoms has still remained largely unknown. It is vitally important to study the fundamentals of gold nanorod growth, because the understanding of this aspect will guide us to newer material design and more sophisticated synthetic methods. In this paper, as a preliminary result in understanding the reaction process of gold atoms, we will report a mechanistic investigation on gold atoms over reaction. Moreover, by successive addition of growth solution to seed solution, the shape and the length of the product could be controlled.

\section{Method}

In a typical synthesis, $0.08 \mathrm{M} \mathrm{CTAB}$ and $250 \mu \mathrm{M} \mathrm{HAuCl}{ }_{4}$ aqueous solution were prepared as the growth solution. The solution was heated to $40{ }^{\circ} \mathrm{C}$ while stirring to dissolve the CTAB. The solution was stored at $27^{\circ} \mathrm{C}$ until cooling to room temperature. Preparation of $\sim 3-4 \mathrm{~nm}$ gold seed particles has already been reported by other researchers, using the trisodium

* To whom correspondence should be addressed. electronic mail: rsliu@ntu.edu.tw.

National Taiwan University.

$\doteqdot$ Hokkaido University.

$\S$ Industrial Technology Research Institute.

"National Synchrotron Radiation Research Center.

$\perp$ National Nano Device Laboratories.
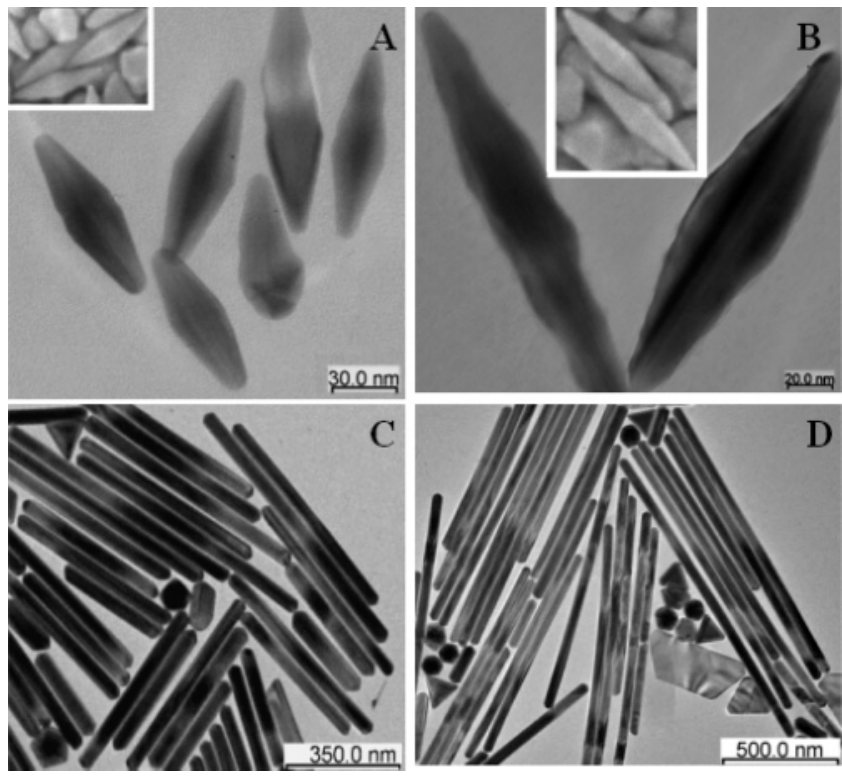

Figure 1. TEM images of gold nanoparticles synthesized by this method. A, B, C, and D represent the seed solutions after first $(0.2$ $\mathrm{mL})$, second $(2 \mathrm{~mL})$, third $(20 \mathrm{~mL})$, and third fourth $(200 \mathrm{~mL})$ additions of growth solution, respectively. The inset shows the SEM image of the corresponding samples.

citrate capping method. ${ }^{3}$ Gold seeds $(0.02 \mathrm{~mL})$ were placed in a beaker. Four quantities $(0.01,0.1,1$, and $10 \mathrm{~mL})$ of freshly prepared AA $(10 \mathrm{mM})$ solutions were mixed with $0.2,2,20$, and $200 \mathrm{~mL}$ of growth solutions, respectively. After AA was added, the growth solution became colorless as gold ions were

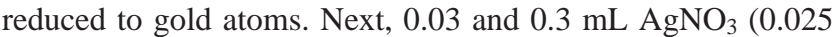
$\mathrm{mM}$ ) solution were added to 0.2 and $2 \mathrm{~mL}$ growth solution, respectively. These four colorless solutions were added to the gold seed solution one by one at $45 \mathrm{~s}$ intervals.

\section{Results and Discussion}

Figure 1 shows a typical transmission electron microscopy (TEM) image of the gold nanoparticles and rods, indicating that 


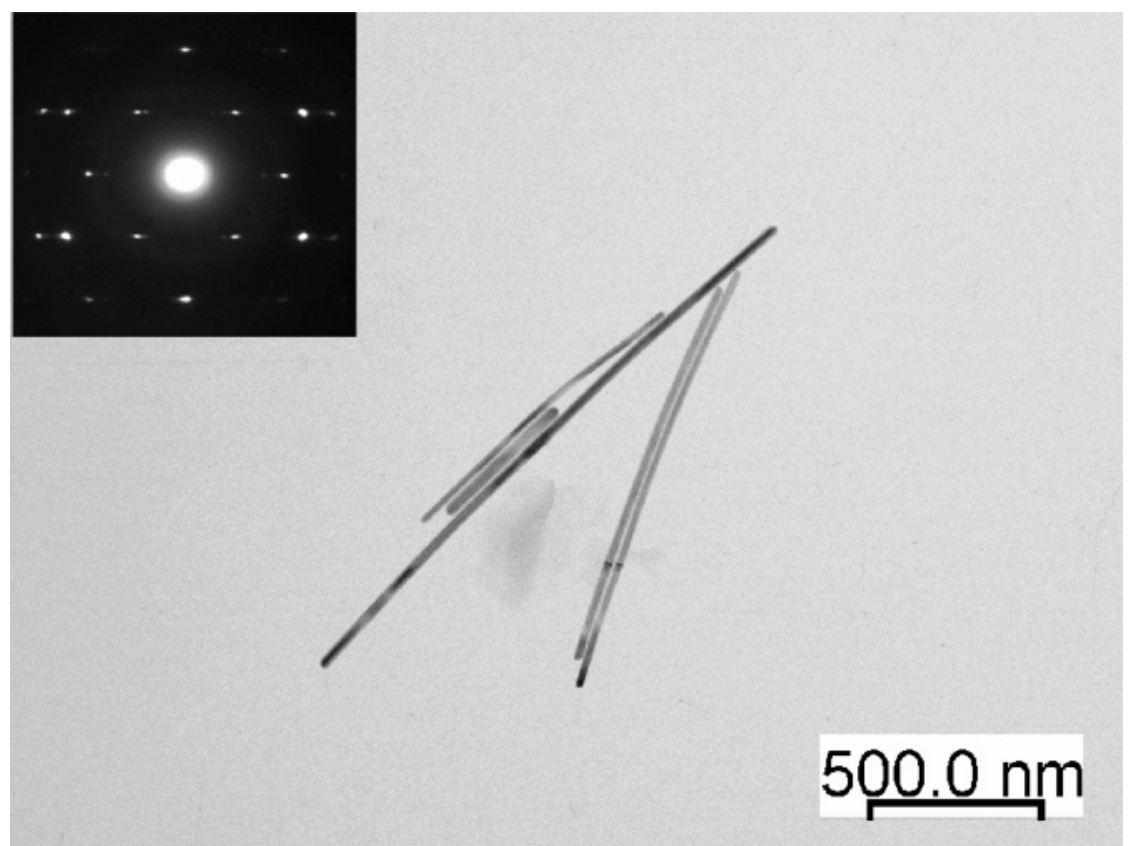

Figure 2. TEM images of nanorods after the fifth growth solution $(2000 \mathrm{~mL})$ was introduced into the seed solution. The inset shows electron diffraction pattern of gold nanowires.

the fusiform nanoparticles have been successfully synthesized as $0.2 \mathrm{~mL}$ (A) and $2 \mathrm{~mL}$ (B) growth solution was added; the inset shows the SEM image of fusiform nanoparticles (Figure 1). In comparison to Figure $1 \mathrm{~A}$ and $\mathrm{B}$, as the third $(20 \mathrm{~mL}$, Figure 1C) and fourth (200 mL, Figure 1D) growth solutions were added, the nanorods could be observed, and the average lengths were $\sim 550 \mathrm{~nm}$ and $1.4 \mu \mathrm{m}$, respectively. Nanorods with aspect ratios of up to $\sim 40$ can be obtained. The TEM and SEM analyses clearly indicate that the shape evolves from fusiform nanoparticles to 1-D rods. Note that the nanorods could be expanded up to $2 \mu \mathrm{m}$ and aspect ratios of nanowires up to 70 (Figure 2; the inset shows the electron diffraction pattern of gold nanowires). Electron diffraction analysis of nanowires shows the superposition of specific crystallographic zones corresponding to the $\langle 110\rangle$ and $\langle 111\rangle$ zones of the face-centered cubic structure, which was consistent with the previous study of nanorods synthesized via the CTAB system. ${ }^{6}$ Murphy and co-workers ${ }^{11}$ established that the amount and the properties of seed particles critically determine the product. The diameter of seeds generally dictates the width of the resulting product. This investigation develops a new method that keeps the [growth solution] $] /[$ seed] ratio constant through the reaction; the addition volume of the growth solution is 10 times that of the last addition. However, the length of the rods cannot be extended by taking a partial solution as seeds from one growth solution to next one, because the [growth solution]/[seed] ratio increased with the further transfers, and seeds introduced into the system were too few to provide enough deposition sites. The rates of nucleation and growth are now widely believed to be dominated by the probabilities of collisions between several atoms, between atoms and clusters, and among two or more clusters. When only a few seeds were introduced into the growth solution, many atoms and few seeds were present in the reaction system, and the probability of an effective collision between atoms exceeded the probabilities of the other two collisions. The collisions of atoms consume many atoms, which form nuclei, so the number of atoms grown on the surface of individual seeds dropped and tended to form small clusters. Consequently, the [growth solution]/[seed] ratio (volume) seemed to play the most important role in determining the final product; the evolution of shape

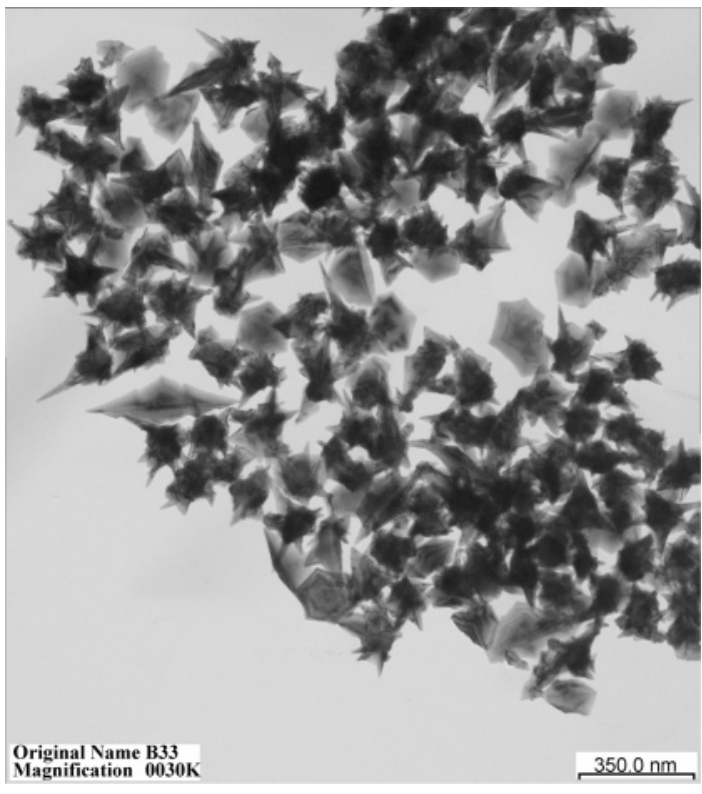

Figure 3. TEM image of branched gold nanoparticles.

strongly depends on the volume of growth solution added. On the other hand, the effect of silver nitrate has been investigated, indicating that the branched gold particles were observed with the existence of silver nitrate. ${ }^{3 a, 9}$ El-Sayed et al. have proposed that silver ions could assist in the template elongation by pairing with $\mathrm{Br}^{-}$ions of CTAB. ${ }^{12}$ Nonetheless, silver nitrate was essential for the formation of fusiform nanoparticles; some spherical or elliptical nanoparticles were observed without the addition of silver nitrate (not shown). The branched gold particles similar to those in a previous study ${ }^{9}$ were found when a substantial amount of silver nitrate was added (Figure 3); the yield of branched particles produced was as high as $\sim 90 \%$. Therefore, the shape and length control has been achieved via varying the addition volume of growth solution.

To understand the optical properties of the fusiform nanoparticles, the absorption spectra are shown in Figure 4. It is well-known that the surface plasmon absorption spectra of gold 


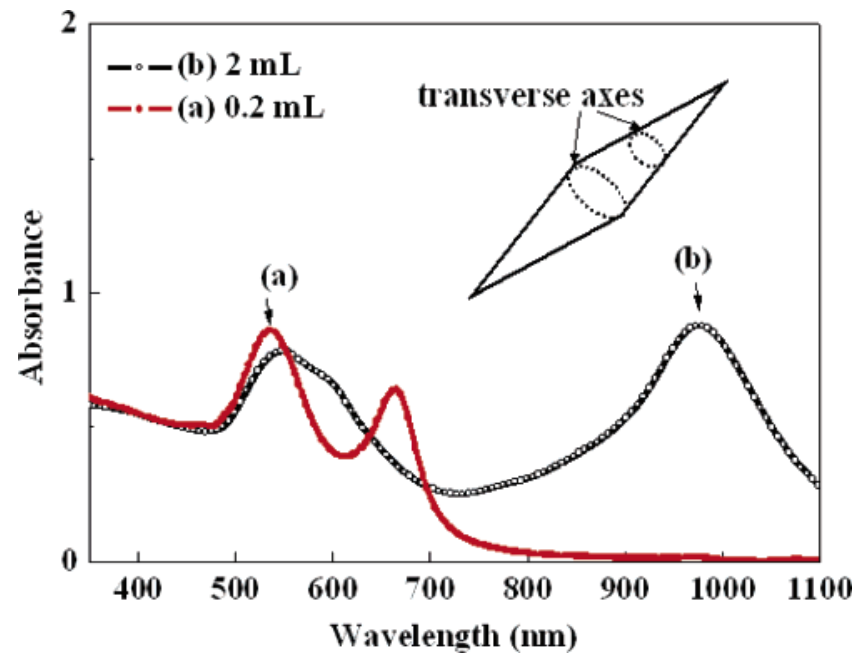

Figure 4. Extinction spectra of Au fusiform nanorods: (a) after first $(0.2 \mathrm{~mL})$ growth solution was added; (b) after second $(2 \mathrm{~mL})$ growth solution was added.

nanorods are characterized by two bands: the shorter wavelength band is attributed to the transverse surface plasmon resonance, and another absorption band appears at longer wavelength, which corresponds to the longitudinal surface plasmon resonance. The fusiform nanoparticles exhibit transverse and longitudinal plasmon bands in the visible region of the spectrum. More specifically, the spectrum of sample (b) displayed two peaks in the transverse band owing to the existence of two transverse axes. However, the transverse band corresponding to sample (a) would not split significantly because of the similar position of two transverse peaks. ${ }^{2}$ Moreover, the longitudinal absorption band exhibits a wider full width at half-maximum (fwhm) with increasing aspect ratio. Since the change of electron density corresponding to the longitudinal mode is gentler than that of the transverse mode, the change of electron density corresponding to the longitudinal mode becomes slower and slower, and then, the fwhm of the longitudinal absorption band increaseswith an increase of aspect ratio. ${ }^{10 \mathrm{~d}}$ Owing to the specific optical properties, it may be applied to related field. Generally, gold nanocluster aqueous suspensions are believed to exhibit strong surface plasmon resonance peaks at $\sim 530 \mathrm{~nm}$, but the red color was not observed, because Au was present in a very small Au nanoparticles surrounded by surfactant molecules. The $\mathrm{CTAB}$ as the capping agent on the surface of the gold nanocluster prevented the aggregation of the nanoclusters to large-sized Au particles. ${ }^{10 a, b, 11 b}$

Figure 1 (Supporting Information) schematically depicts the possible steps in the formation of 1-D nanorods in the present method. The role of the surfactant is to constrain the particle growth in one dimension and stabilize the resultant product. ${ }^{10 \mathrm{a}-\mathrm{c}}$ It causes the morphology of the nanoclusters to evolve from fusiform into 1-D nanorods. Therefore, the nanorods' shape and length could be controlled.

\section{Conclusions}

In conclusion, the TEM images show the change in the morphology of gold nanoparticles during the reaction: the shape of the gold nanoparticles evolved from fusiform into 1-D rods as more growth solution was added. Accordingly, a simple route for the large-scale synthesis of 1-D nanorods of gold, whose length and shape is controllable by varying the amount of growth solution, was demonstrated. These particles are expected to have wide applications in sensing and other related fields.

Acknowledgment. We would like to thank the National Science Council of the Republic of China, Taiwan, for financially supporting this research under contract no. NSC 942113-M-002-030.

Supporting Information Available: The reaction mechanism. This material is available free of charge via the Internet at http://pubs.acs.org.

\section{References and Notes}

(1) (a) Murphy, C. J. Science 2002, 298, 2139. (b) Hu, J.; Odom, T. W.; Lieber, C. M. Acc. Chem. Res. 1999, 32, 435. (c) El-Sayed, M. A. Acc. Chem. Res. 2001, 34, 257. (d) Huang, M. H.; Mao, S.; Feick, H.; Yan, H.; Wu, Y.; Kind, H.; Weber, E.; Russo, R.; Yang, P. Science 2001 292, 1897. (e) Huynh, W. U.; Dittmer, J. J.; Alivisatos, A. P. Science 2002 295,2425

(2) (a) Yu, Y.-Y.; Chang, S.-S.; Lee, C.-L.; Wang, C. R. C. J. Phys. Chem. B 1997, 101, 6661. (b) Wilson, O.; Wilson, G. J.; Mulvaney, P. Adv. Mater. 2002, 14, 1000. (c) Favier, F.; Walter, E.; Zach, M. P.; Benter, T.; Penner, R. M. Science 2001, 293, 2227. (d) Maier, S. A.; Kik, P. G.; Atwater, H. A.; Meltzer, S.; Harel, E.; Koel, B. E.; Requicha, A. A. G. Nat. Mater. 2003, 2, 229.

(3) (a) Jana, N. R.; Gearheart, L.; Murphy, C. J. Adv. Mater. 2001, 13, 1389. (b) Jana, N. R.; Gearheart, L.; Murphy, C. J. J. Phys. Chem. B 2001, 105, 4065 .

(4) (a)Sun, Y.; Gates, B.; Mayers, B.; Xia, Y. Nano Lett. 2002, 2, 165. (b) Zhang, Z.; Zhao, B.; Hu, L. J. Solid State Chem. 1996, 121, 105. (c) Jana, N. R.; Gearheart, L.; Murphy, C. J. Chem. Commun. 2001, 617. (d) Caswell, K. K.; Bender, C. M.; Murphy, C. J. Nano Lett. 2003, 3, 667.

(5) Chang, S.-S.; Shih, C.-W.; Chen, C.-D.; Lai, W.-C.; Wang, C. R. C. J. Phys. Chem. B 1999, 15, 701.

(6) Johnson, C. J.; Dujardin, E.; Davis, S. A.; Murphy, C. J.; Mann, S. J. Mater. Chem. 2002, 12, 1765.

(7) (a) Tornblom, M.; Henriksson, U. J. Phys. Chem. B 1997, 101 6028. (b) Mohamed, M. B.; Ismail, K. Z.; Link, S.; El-Sayed, M. A. J. Phys. Chem. B 1998, 102, 9370.

(8) (a) Jana, N. R.; Gearheart, L.; Obare, S. O.; Murphy, C. J. Langmuir 2002, 18, 922. (b) Chang, S.-S.; Shih, C.-W.; Chen, C. D.; Lai, W. C.; Wang, C. R. C. Langmuir 1999, 15, 701. (c) Link, S.; Mohamed, M. B.; El-Sayed, M. A. J. Phys. Chem. B 1998, 103, 3073.

(9) Sau, T. K.; Murphy, C. J. J. Am. Chem. Soc. 2004, 126, 8648.

(10) (a) Gao, J.; Bender, C. M.; Murphy, C. J. Langmuir 2003, 19, 9065. (b) Busbee, B. D.; Obare, S. O.; Murphy, C. J. Adv. Mater. 2003, 15, 414. (c) Nikoobakht, B.; El-Sayed, M. A. Langmuir 2001, 17, 6368. (d) Zhu, J. Phys. Lett. A 2005, 339, 466.

(11) (a) Sau, T. K.; Murphy, C. J. Langmuir 2004, 20, 6414. (b) Gole, A.; Murphy, C. J. Chem. Mater. 2004, 16, 3633.

(12) Nikoobakht. B.; El-Sayed, M. A. Chem. Mater. 2003, 15, 1957. 